\title{
CT screening for lung cancer brings forward early disease. The randomised Danish Lung Cancer Screening Trial: status after five annual screening rounds with low-dose CT
}

\author{
Zaigham Saghir, ${ }^{1}$ Asger Dirksen, ${ }^{1}$ Haseem Ashraf, ${ }^{2}$ Karen Skjøldstrup Bach, ${ }^{3}$ \\ John Brodersen, ${ }^{4}$ Paul Frost Clementsen, ${ }^{1}$ Martin Døssing, ${ }^{5}$ Hanne Hansen, ${ }^{6}$ \\ Klaus Fuglsang Kofoed, ${ }^{7}$ Klaus Richter Larsen, ${ }^{8}$ Jann Mortensen, ${ }^{9}$ \\ Jakob Fraes Rasmussen, ${ }^{4}$ Niels Seersholm, ${ }^{1}$ Birgit Guldhammer Skov, ${ }^{10}$ Hanne Thorsen, ${ }^{4}$ \\ Philip Tønnesen, ${ }^{1}$ Jesper Holst Pedersen ${ }^{11}$
}

\section{See Editorial, p 283}

1 Department of Respiratory Medicine, Gentofte University Hospital, Hellerup, Denmark ${ }^{2}$ Department of Radiology, Akershus University Hospital, Lørenskog, Norway

${ }^{3}$ Department of Radiology, Gentofte University Hospital Hellerup, Denmark

${ }^{4}$ The Research Unit and Section for General Practice, Department of Public Health, Faculty of Health Sciences, University of Copenhagen, Copenhagen, Denmark

${ }^{5}$ Department of Internal Medicine, Nordsjælland University Hospital, Frederikssund, Denmark ${ }^{6}$ Department of Radiology, Bispebjerg University Hospital, Copenhagen, Denmark ${ }^{7}$ Department of Cardiology, Copenhagen University Hospital, Copenhagen, Denmark ${ }^{8}$ Department of Respiratory Medicine, Bispebjerg University Hospital, Copenhagen, Denmark ${ }^{9}$ Department of Clinical Physiology and Nuclear Medicine, Copenhagen University Hospital, Copenhagen, Denmark ${ }^{10}$ Department of Pathology, Bispebjerg University Hospital, Copenhagen, Denmark ${ }^{11}$ Department of Thoracic Surgery, Copenhagen University Hospital, Copenhagen, Denmark

\section{Correspondence to}

Dr Zaigham Saghir, Department of Respiratory Medicine, Gentofte University Hospital, Niels Andersens Vej 65, Opgang 30A, 2900 Hellerup, Denmark; zaigham.saghir@gmail.com

Received 3 July 2011 Accepted 14 December 2011 Published Online First 27 January 2012

\section{ABSTRACT}

Background The effects of low-dose CT screening on disease stage shift, mortality and overdiagnosis are unclear. Lung cancer findings and mortality rates are reported at the end of screening in the Danish Lung Cancer Screening Trial.

Methods 4104 men and women, healthy heavy smokers/former smokers were randomised to five annual low-dose CT screenings or no screening. Two experienced chest radiologists read all CT scans and registered the location, size and morphology of nodules. Nodules between 5 and $15 \mathrm{~mm}$ without benign characteristics were rescanned after 3 months. Growing nodules ( $>25 \%$ volume increase and/or volume doubling time $<400$ days) and nodules $>15 \mathrm{~mm}$ were referred for diagnostic workup. In the control group, lung cancers were diagnosed and treated outside the study by the usual clinical practice.

Results Participation rates were high in both groups (screening: 95.5\%; control: 93.0\%; $p<0.001$ ). Lung cancer detection rate was $0.83 \%$ at baseline and mean annual detection rate was $0.67 \%$ at incidence rounds $(p=0.535)$. More lung cancers were diagnosed in the screening group (69 vs $24, p<0.001$ ), and more were low stage (48 vs 21 stage I-IIB non-small cell lung cancer (NSCLC) and limited stage small cell lung cancer ( $S C L C), p=0.002$ ), whereas frequencies of high-stage lung cancer were the same (21 vs 16 stage IIIA-IV NSCLC and extensive stage SCLC, $p=0.509$ ). At the end of screening, 61 patients died in the screening group and 42 in the control group $(p=0.059) .15$ and 11 died of lung cancer, respectively $(p=0.428)$.

Conclusion CT screening for lung cancer brings forward early disease, and at this point no stage shift or reduction in mortality was observed. More lung cancers were diagnosed in the screening group, indicating some degree of overdiagnosis and need for longer follow-up.

\section{INTRODUCTION}

In general, the purpose of screening for cancer is detecting the disease at a curable stage. Previous lung cancer screening studies have found a high frequency of early stage cancer compared with

\section{Key messages}

What is the key question?

- To report lung cancer findings, disease stage shift, mortality rates at the end of screening in the Danish Lung Cancer Screening Trial.

What is the bottom line?

- With screening, low-stage lung cancers are diagnosed more frequently but at this point no stage shift is observed and pooling of mortality data with European screening trials is needed.

\section{Why read on?}

- The importance of stage shift in a screening setting and overdiagnosis is discussed from the results presented.

patients with symptomatic lung cancer. ${ }^{1-4}$ However, a high frequency of early stage cancers is not advantageous in itself. Experiences from other cancer screening programmes have indicated that incidence rate of early disease increased substantially with screening, while reduction in number of advanced cancers was negligible. This meant a decrease in relative proportion (relative stage shift) of advanced cancers but not in absolute numbers (absolute stage shift). ${ }^{5-7} \mathrm{~A}$ high frequency of early stage cancers is only beneficial if at the same time the frequency of late stage cancers is reduced, implying a reduction in mortality. Alternatively, a high frequency of early stage cancer may be due to 'over-diagnosis', that is, cancer that never would have progressed to clinical disease during a person's lifetime, and thus would not have been diagnosed without screening. Overdiagnosis is potentially harmful because it may imply unnecessary investigations and treatment. At present it is not clear to what extent the high frequencies of early stage lung cancer in screening trials are due to overdiagnosis and the degree to which they indicate a beneficial stage shift and reduced mortality.

In this paper, we report the lung cancer findings after five annual screening rounds in the 
randomised Danish Lung Cancer Screening Trial (DLCST). This includes the prevalence of lung nodules, stage and histology of lung cancers. We also report lung cancer specific mortality and all-cause mortality at the end of the 4-year screening period.

\section{MATERIAL AND METHODS Study population}

The overall design and baseline results of DLCST have previously been published. ${ }^{8} 9$

DLCST is a 5-year prospective randomised controlled screening trial. From October 2004 to March 2006, 4104 men and women were enrolled after written informed consent was acquired. They all volunteered after reading advertisements in local and regional free newspapers. Ads contained information about general study design, enrolment criteria and governmental funding.

We included men and women aged 50-70 years, who were current or former smokers with at least 20 pack years of smoking history. Former smokers should have quit after the age of 50 years and be abstinent for $<10$ years. Participants had to be able to climb two flights of stairs (36 steps) without pausing. Lung function was measured by spirometry and forced expiratory volume in the first second had to be at least $30 \%$ of predicted.

Exclusion criteria were weight over $130 \mathrm{~kg}$, history of cancer diagnosis and treatment, lung tuberculosis, illness that would shorten life expectancy to $<10$ years and chest CT received during the last year for any reason.

After inclusion, the participants were randomised to the screening group $(n=2052)$ or the control group $(n=2052)$. The screening group received five annual low-dose chest CT scans (one baseline scan and four subsequent incidence scans). All participants had an annual visit at the screening clinic, where lung function tests were performed, and questionnaires concerning health, lifestyle, smoking habits and psychosocial consequences of screening were completed. ${ }^{10-12}$ Figure 1 shows an overview of the DLCST design.

\section{Imaging and image review}

Scanning procedures have been described previously. ${ }^{8}$ Two experienced chest radiologists $(\mathrm{KB}$ and $\mathrm{HH})$ read all $\mathrm{CT}$ scans and registered the location, size and morphology of nodules. Scans with no nodules or nodules $<5 \mathrm{~mm}$ (category 2) or nodules up to $15 \mathrm{~mm}$ in diameter with benign characteristics (category 1) were screening test negative. Participants with nodules with a diameter between 5 and $15 \mathrm{~mm}$ without benign characteristics (category 3) were rescanned after 3 months. Participants with nodules larger than $15 \mathrm{~mm}$ (category 4) or rapid growing nodules ( $>25 \%$ increase in volume) (category 5 ) were referred to chest physicians for diagnostic workup. Siemens syngo LungCARE CT version VE25A, a commercial computer-aided detection software, was used to measure nodule volumes when possible, and volume doubling time (VDT) was used to measure growth rate and was considered a supplement in decision making. Rapid growth (VDT <400 days) increased the suspicion of malignancy, whereas slow growth (VDT >400 days) decreased suspicion. The same software was used in the DutchBelgian screening trial $(\mathrm{NELSON})^{13}$ and was validated in a double reading project. ${ }^{14}$

Findings were discussed at weekly consultations with participation of radiologists ( $\mathrm{KSB}, \mathrm{HH})$, pulmonologists ( $\mathrm{AD}, \mathrm{PFC}$ ) and a chest surgeon (JHP), and decisions were recorded in the project database.

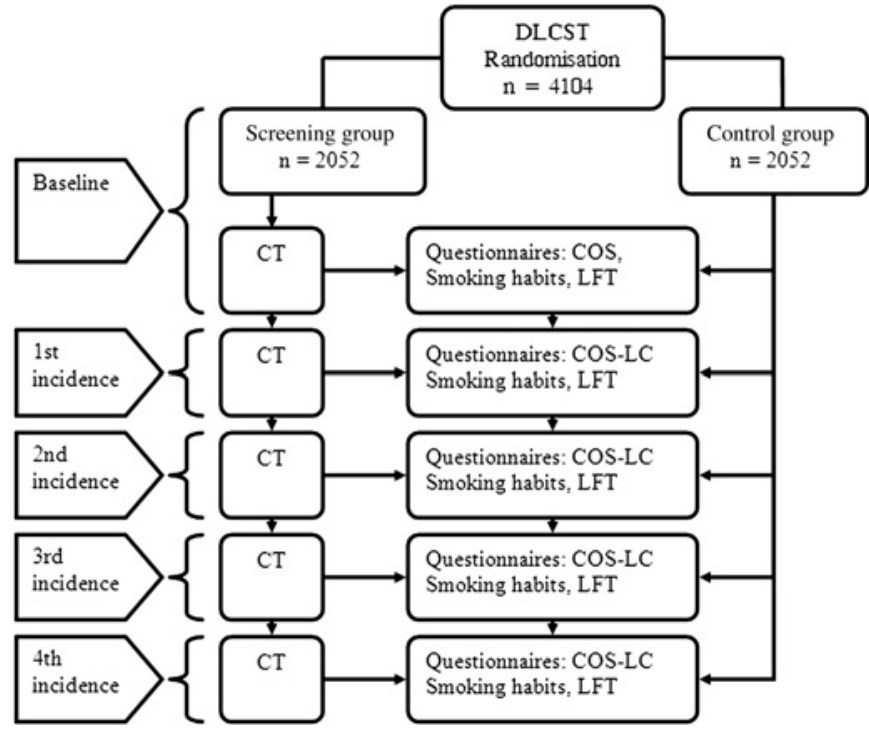

Figure 1 Overall design of the Danish Lung Cancer Screening Trial (DLCST). COS, consequences of screening ${ }^{10}$; COS-LC, consequences of screening in lung cancer ${ }^{11} ; \mathrm{CT}$, low-dose multi-slice computer tomography; LFT, lung function test. ${ }^{12}$

\section{Diagnostic workup}

Participants with positive screening findings who needed further diagnostic workup were referred to chest physicians in two specialised lung cancer diagnostic centres at Gentofte University Hospital and Bispebjerg University Hospital in Copenhagen. An individual diagnostic strategy based on the characteristics of the radiological findings was followed with the possibility of CT with intravenous contrast, ${ }^{18} \mathrm{~F}$-fluorodeoxyglucosepositron-emission-tomography-CT (PET-CT), bronchoscopy with biopsies, endobronchial ultrasound and endo-oesophageal ultrasound, CT-guided transthoracic biopsy and mediastinoscopy. If necessary, video-assisted thoracoscopic surgery was performed to obtain a final diagnosis and definitive treatment was sometimes performed in the same procedure. This was in accordance with European guidelines. Final staging of disease was made according to the cytology/histology of the cancers (pTNM) and the recommendations from the International Association for the Study of Lung Cancer (IASLC) seventh edition. ${ }^{15}$ Pathology was performed by BGS according to the EUUS pathology panel guidelines for screening detected lung tumours and subsequently confirmed by the panel. ${ }^{8}$

In the control group, lung cancers were diagnosed and treated outside the study by the usual clinical practice, which mostly involved the same lung cancer diagnostic centres and the same diagnostic and treatment strategy as for the screening group.

\section{Follow-up}

Both randomisation groups were followed annually at study visits, where information on imaging, history of diagnostic workup and treatment was gathered. Annual inquiries to the Danish Lung Cancer Register were made, and lung cancer data were included in the database. Subsequently relevant medical journals were retrieved from diagnosing and treating departments.

Mortality information was obtained annually from the Danish Civil Registration System, which contains information on all national deaths with a delay of a couple of weeks. Cause of death information was extracted from the Danish Causes of Death Register, which has a lag time in registry update of up to 2 years. Therefore, in addition to the registry inquiries, the 
medical history of the deceased was obtained, if possible, from general practitioners, medical records of hospitals, autopsy reports, police departments and the National Board of Health. The cause of death was established by a local death review board and categorised as 'lung cancer' or 'other causes' of death.

In this paper, the latest follow-up for both groups was set as end of screening, 31 March 2010. Participants who emigrated from Denmark were lost to follow-up.

\section{Data analysis}

$\chi^{2}$ test was used to compare frequencies of disease and participation rates between the screening and control group. Two sample t test was used to test the null hypothesis comparing means of follow-up years in the two groups. The log-rank test and Kaplan-Meier plot were used to assess mortality rates. We used a $95 \% \mathrm{CI}$ and $\mathrm{p}$ values below 0.05 were considered statistically significant.

Power calculation was made assuming pooling of mortality data with the NELSON study. Assuming a 1:1 randomisation, a power of $80 \%$, significance level of $5 \%$, an annual incidence of lung cancer of $0.5 \%$, a difference in 5 -year mortality between screened and non-screened participants of at least 20\%, 95\% compliance in the screen group, $5 \%$ contamination in the control group, and 10 years of follow-up after randomisation, the required sample size was calculated as $2 \times 2000$ participants.

$\mathrm{R}$ statistical software version 2.12 .1 was used as the statistical tool.

\section{Ethical and legal approval}

DLCST was approved by the Ethics Committee of Copenhagen County and the Danish Data Protection Agency, and registered in Clinical Trials.gov Protocol Registration System (identification no. NCT00496977).

\section{RESULTS \\ Participation}

The baseline characteristics of all participants have been previously published. ${ }^{8}$ No statistical difference in age, gender, lung function, smoking status and pack-years was found in the two randomisation groups. The last screening scan was performed in March 2010.

The mean annual participation rate in the screening group was $95.5 \%(2047+1976+1944+1982+1851=9800) /(2052 \times 5)$, and in the control group the mean rate of participation in the annual study visits was $93.0 \% \quad(2052+1953+1877+1838$ $+1820=9540) /(2052 \times 5)$.

The difference in participation rate between the two groups was significant in second and third incidence rounds $\left(\chi^{2}\right.$ test: $\mathrm{p}<0.001)$. No significant difference was found at baseline, and the first and fourth incidence rounds $\left(\chi^{2}\right.$ test: $p=0.073 ; 0.089$; $0.127)$.

Total person-years of follow-up at last screening were 9769 person-years in the screening group; median 4.81, mean 4.76 (95\% CI 3.64 to 5.88) and 9794 person-years in the control group, median 4.81, mean 4.77 ( $95 \%$ CI 3.73 to 5.82 ). No difference was seen between the groups (two sample t test; $\mathrm{p}=0.479$ ) Twenty-nine participants were lost to follow-up due to emigration. Fifteen were in the screening group, and all screened negative at their last CT scan.

Radiological findings and management of nodules in the CT arm During five screening rounds, 1029 (560 baseline +469 incidence) non-calcified nodules (category 2-4) were registered 1404 times in 611 participants. Table 1 shows the categories of nodules detected during the five screening rounds in relation to the number of persons screened. Compared with baseline, the frequency of category 2-4 nodules was considerably lower during the incidence rounds $\left(\chi^{2}\right.$ test: $\left.p<0.001\right)$.

At baseline recall rate for follow-up scan was 7.6\% (155/2047). During the following years the rates were $20(1.0 \%), 24$ $(1.2 \%), 18(0.9 \%)$ and $24(1.3 \%)$. A total of 198 of $9800(2.02 \%)$ participants were referred for diagnostic evaluation by pulmonologists. Seven participants of $9800(0.07 \%)$ had a diagnostic video-assisted thoracoscopic surgery procedure for benign disease. We plan to publish the types and extent of procedures and the economic costs separately. The diagnostic false positive rate at baseline was $7.9 \%,{ }^{8}$ but at subsequent screening rounds the rates were $34(1.7 \%), 39(2.0 \%), 32(1.6 \%), 35(1.9 \%)$.

\section{Lung cancers}

The disease stages for both randomisation groups are presented in table 2 . The baseline cancers have previously been reported $^{8}$ and are updated according to IASLC classification recommendations. ${ }^{15}$

\section{Screening group}

A total of 69 lung cancers were diagnosed. The overall detection rate (baseline + incidence rounds) was $0.70 \%$ (69 of 9800). At baseline the detection rate was $0.83 \%$, while the incidence rounds had a mean annual detection rate of $0.67 \%$ ( $\chi^{2}$ test; $\mathrm{p}=0.535)$. Three were small cell lung cancers (SCLCs) and 66 were non-small cell lung cancers (NSCLCs). Forty-eight (70\%) were early stage (I-IIB NSCLC and limited stage SCLC) and thus potentially curable. Twenty-two $(30 \%)$ were diagnosed in late stage (IIIA-IV NSCLC and extensive stage SCLC). Fiftyeight $(85 \%)$ of the lung cancers were pathologically verified within a year from the CT scan when they were first detected.

One interval cancer was diagnosed 10 months after the third incidence scan when a 65-year-old man complained of hip pain, and he was admitted to the hospital where chest CT and skin biopsy showed disseminated adenocarcinoma originating from the lungs (CK7, TTF-1, CDX2 positive; CK5/6, CK20, PSA negative). He received palliative chemotherapy and radiation and died a month later. Even in retrospect, no primary focus could be identified in the lungs by visual inspection of the four previous screening scans.

\section{Control group}

Twenty-four participants were diagnosed with lung cancer. Six had extensive stage SCLC and one limited stage. Eight (33\%) were in early stage and $16(67 \%)$ in late stage. One of the participants with an early stage lung cancer had received a CT scan for screening purposes via the general practitioner (contamination). A stage IA lung cancer was found and was treated by surgery.

Significantly more lung cancers were diagnosed in the screening group ( $\chi^{2}$ test: 69 vs $\left.24 ; p<0.001\right)$ and more were low

Table 1 Detection of non-calcified nodules during five annual screening rounds in 611 participants in the Danish Lung Cancer Screening Trial

\begin{tabular}{llllllr}
\hline Nodules category & Baseline & 1st year & 2nd year & 3rd year & 4th year & Total \\
\hline $2(<5 \mathrm{~mm})$ & 371 & 185 & 102 & 154 & 80 & 892 \\
$3(5-15 \mathrm{~mm})$ & 151 & 99 & 38 & 51 & 46 & 385 \\
$4(>15 \mathrm{~mm})$ & 38 & 18 & 14 & 31 & 26 & 127 \\
Total & 560 & 302 & 154 & 236 & 152 & 1404 \\
() & $(160)$ & $(94)$ & $(157)$ & $(58)$ & $(469)$ \\
\hline
\end{tabular}


Table 2 Disease stages in the Danish Lung Cancer Screening Trial after five annual CT scans and study visits

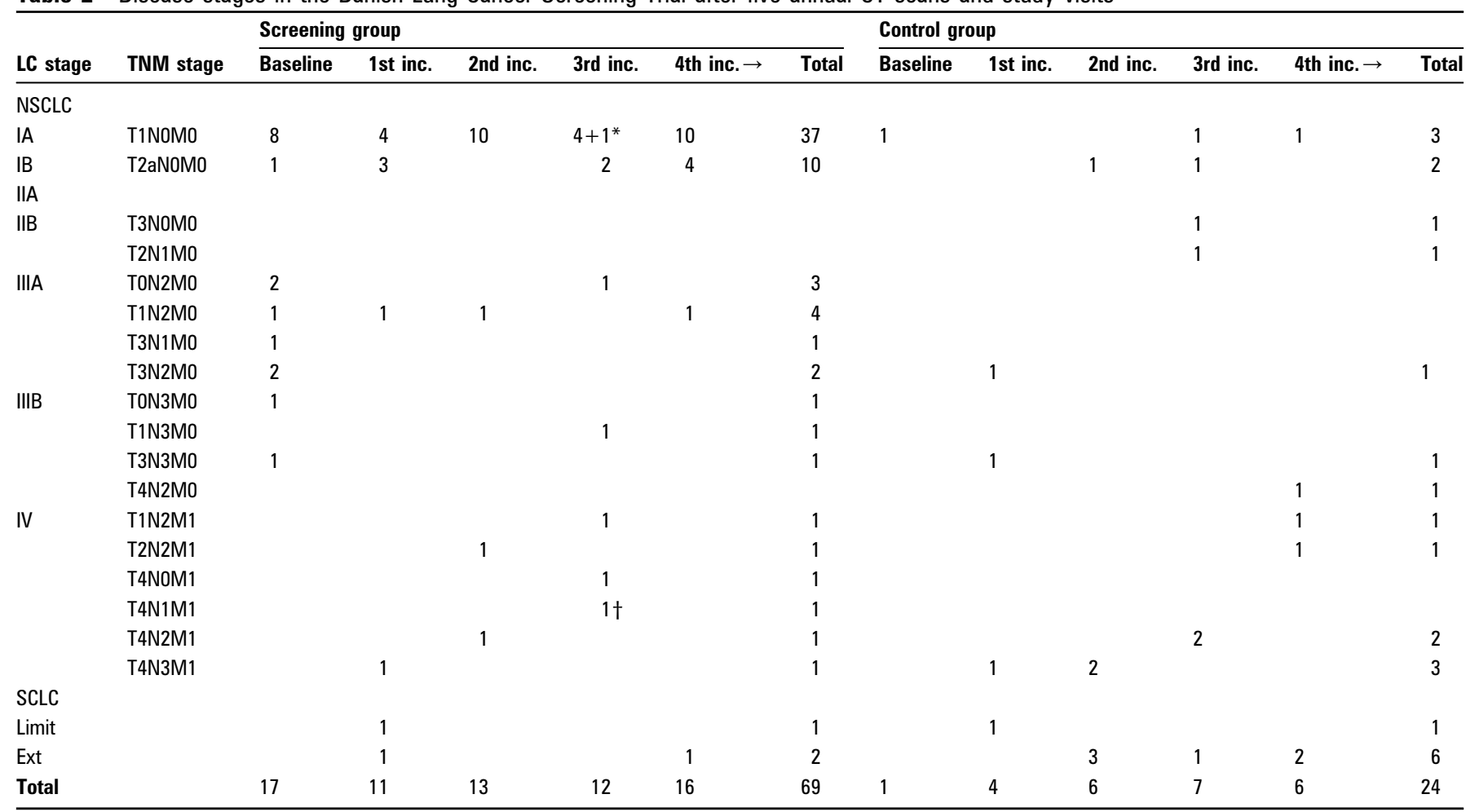

$\rightarrow$, Diagnosed until end of screening.

*Diagnosed as second cancer in patient who also had a stage IB cancer.

tInterval cancer.

Ext, extensive; Inc., incidence; LC, lung cancer; Limit, limited; NSCLC, non-small cell lung cancer; SCLC, small cell lung cancer.

stage ( $\chi^{2}$ test: 48 vs $\left.21 ; p=0.002\right)$. The number of late stage lung cancers was the same in both groups ( $\chi^{2}$ test: 21 vs 16; $p=0.509)$. The absolute and relative stage distributions are shown in figure 2.

The number of new lung cancers remained consistently high during all four incidence rounds in the screened group compared with the control group: $11+13+12+16$ vs $4+6+7+6$ ( $\chi^{2}$ test $\mathrm{p}=0.001)$. Although the number of SCLCs was low in both groups (three vs seven), there was a tendency towards more advanced disease in the control group.

The majority of lung cancers in both study arms were adenocarcinomas. The histology distribution of lung cancers in the screening and control group is shown in table 3.
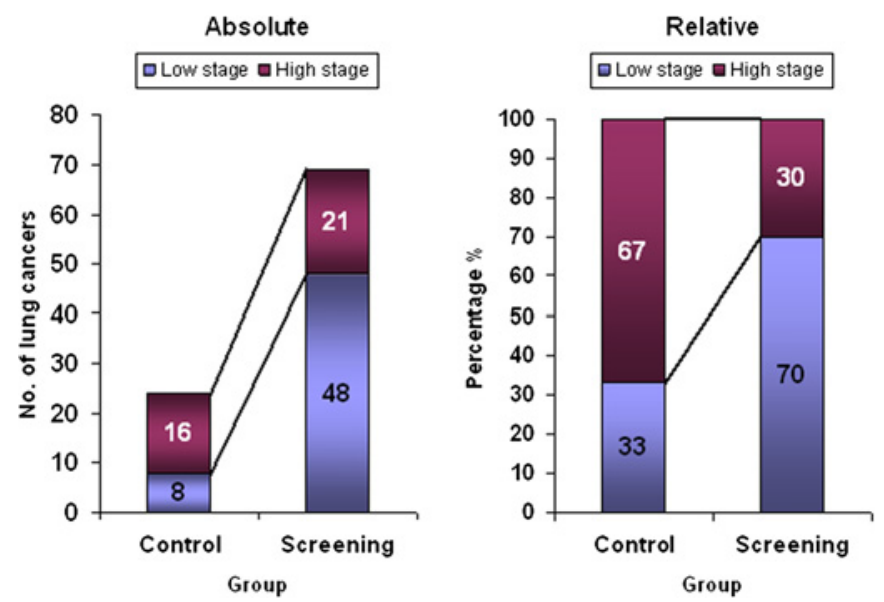

Figure 2 Overview of relative and absolute distribution of lung cancer stage.

\section{Mortality}

According to the Danish Civil Registration system, at the end of screening on 31 March 2010, 103 of 4104 participants had died. Sixty-one (2.97\%) died in the screening group, while $42(2.05 \%)$ died in the control group (log-rank test, all-cause mortality: $\mathrm{p}=0.059)$. Fifteen $(0.73 \%)$ died of lung cancer in the screening group compared with $11(0.54 \%)$ in the control group (log-rank test, lung cancer specific mortality: $p=0.428$ ). Thus, no differences in mortality were found. Figure 3 shows Kaplan-Meier plots of lung cancer and all-cause mortality.

\section{DISCUSSION}

Lung cancer is by far the most lethal cancer type in the world with approximately 1.4 million deaths in 2008 according to

Table 3 Histology of lung cancers in the Danish Lung Cancer Screening Trial

\begin{tabular}{lcc}
\hline Histology & Screening group & Control group \\
\hline NSCLC & 6 & 3 \\
ACL & 34 & 8 \\
ACL + BAC & 4 & \\
ACL mixed subtype & 8 & 2 \\
ACL + SOC & 1 & \\
ACL + LCLC & 1 & 3 \\
SOC & 7 & \\
BAC & 2 & 1 \\
LCLC & 3 & 5 \\
SCLC & 3 & 2 \\
Combined SCLC & & 24 \\
Total & 69 &
\end{tabular}

ACL, adenocarcinoma; BAC, broncho-alveolar carcinoma; LCLC, large cell lung carcinoma; NSCLC, non-small cell lung carcinoma; SCLC, small cell lung carcinoma; SOC, squamous cell carcinoma. 

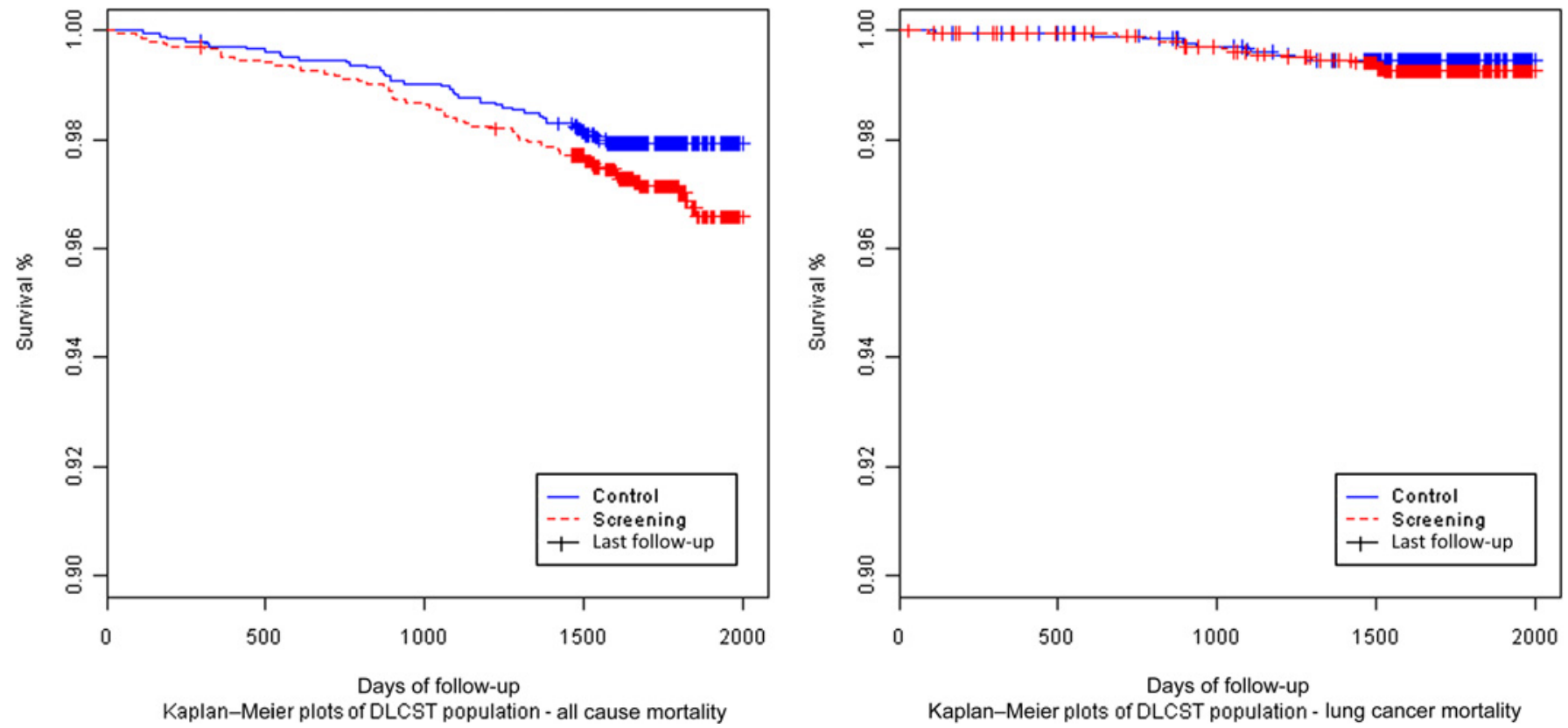

Figure 3 Kaplan-Meier plots of lung cancer and all-cause mortality. DLCST, Danish Lung Cancer Screening Trial.

WHO. ${ }^{16}$ Several efforts have been made to reduce lung cancer mortality. Prevention of risk factors, increased public awareness of symptoms, and early detection and treatment seem to be the cornerstones in the strategy against continuous high mortality.

Lung cancer screening may be a valuable tool in this strategy. However, psycho-social consequences, overdiagnosis, potentially harmful procedures and overtreatment are important drawbacks.

In this paper, we have reported the findings after five annual CT screenings in the DLCST. Participation rates were high in both groups throughout the trial. The rates are lower in the control group, which is probably due to disappointment of not getting screened. Despite this, the extent of contamination, defined as screening in the control group, was very limited in DLCST. ${ }^{9}$

The low recall and false positive rates observed in this study are comparable with those observed in the NELSON trial. ${ }^{13}$ This indicates that the very high recall rates observed in the National Lung Screening Trial (NLST) trial ${ }^{17}$ may be reduced and thereby one of the main obstacles to the implementation of CT screening may be overcome.

The number of early stage lung cancers was six times higher in the screening group compared with the control group. Early stage cancers were defined as stage I-IIB and were potentially curable with surgery alone or with adjuvant chemotherapy. The relative proportion of late stage cancers in the screening group was considerably lower than in the control group. However, no significant difference was seen in the absolute numbers of late stage cancers in both groups.

This suggests the absence of an absolute stage shift, and the excess proportion of early stage cancers (37\%) may indicate some degree of overdiagnosis. Assuming that lung cancer arises with the same frequency in both groups after baseline, the consistently larger numbers of new cancers in the screening group at incidence rounds compared with the control group may indicate a considerable degree of overdiagnosis or a longer lead time until clinical diagnosis in the control group is made and registered. However, some of this overdiagnosis may be due to lag in registration. Information on lung cancer in the control group is not as up to date as in the screening group. Late onset of symptoms and diagnosis may be apparent during the following years and lead to a higher number of late stage cancers in the control group. Therefore, follow-up after several years may change the stage distribution, indicating an absolute stage shift and a subsequent increase in mortality, and also further clarify the extent of overdiagnosis.

Recently, colleagues from the NLST ${ }^{18}$ published their results and found a $20 \%$ lung cancer mortality reduction and $7 \%$ reduction in all-cause mortality. ${ }^{17}$ The NLST randomised 53454 participants and compared low-dose chest CT with chest radiography. The screening began in 2002 and completed in 2006.

After five screening rounds, the mortality data in the DLCST showed no difference in lung cancer or all-cause mortality. This is explained by the small sample size and shorter follow-up time. However, we expect that a possible screening effect on mortality may be apparent during the next $4-5$ years when the follow-up time will be approximately the same as in NLST. If screening reduces mortality, one should expect that the screened participants will eventually have fewer incurable high-stage lung cancers than the non-screened population. According to IASLC, survival data for high-stage lung cancers (pathologic stage IIIB-IV) show a median survival time of 13-17 months. ${ }^{15}$ Thus, in principle, a stage shift should precede mortality reduction with 1-2 years only. However, as lung cancer growth may show great heterogeneity, lead time may be much longer and perhaps even up to $4-5$ years. ${ }^{19}$

To gain more statistical power, efforts are being made to pool all mortality data in the European randomised trials. ${ }^{2} 1320$ These efforts are challenging due to differences in study design and time span. However, it is our hope that within the next decade, data from DLCST together with data from other randomised European trials will substantially improve our knowledge on potential harms and benefits of lung cancer screening with low-dose CT.

Funding This study was supported by the Danish Ministry of Interior and Health.

Competing interests None.

Ethics approval Ethics approval was provided by Committee of Copenhagen County and the Danish Data Protection Agency. 
Contributors Steering Committee (initiated trial design, data collection and statistical analysis plan): Jesper Holst Pedersen (Chairman/Pl/Guarantor), Asger Dirksen (Guarantor), Karen Skjøldstrup Bach, John Brodersen, Paul Frost Clementsen, Martin Døssing, Hanne Hansen, Klaus Fuglsang Kofoed, Klaus Richter Larsen, Jann Mortensen, Niels Seersholm, Birgit Guldhammer Skov, Hanne Thorsen, Philip Tønnesen, Haseem Ashraf (2007) and Zaigham Saghir (2009/Guarantor). HH and KSB performed reading of CT scans. AD, PFC, KRL, JM, NS, BGS, PT conducted diagnostic workup. $A D$ was responsible of event monitoring and following protocol. JB and HT designed the COS and COS-LC questionnaires. ZS and HA collected, cleaned and analysed data. JFR collected data. ZS drafted and revised the paper. All revised the paper.

Provenance and peer review Not commissioned; externally peer reviewed.

\section{REFERENCES}

1. Henschke Cl, McCauley DI, Yankelevitz DF, et al. Early Lung Cancer Action Project: overall design and findings from baseline screening. Lancet 1999;354:99-105

2 Infante M, Cavuto S, Lutman FR, et al. A randomized study of lung cancer screening with spiral computed tomography: three-year results from the DANTE trial. Am J Respir Crit Care Med 2009;180:445-53.

3. Sone S, Takashima S, Li F, et al. Mass screening for lung cancer with mobile spiral computed tomography scanner. Lancet 1998;351:1242-5.

4. Swensen SJ, Jett JR, Hartman TE, et al. CT screening for lung cancer: five-year prospective experience. Radiology 2005;235:259-65.

5. Zahl PH, Maehlen J, Welch HG. The natural history of invasive breast cancers detected by screening mammography. Arch Intern Med 2008;168:2311-16.

6. Welch HG, Woloshin S, Schwartz LM, et al. Overstating the evidence for lung cancer screening: the International Early Lung Cancer Action Program (I-ELCAP) study. Arch Intern Med 2007:167:2289-95.

7. Esserman L, Shieh Y, Thompson I. Rethinking screening for breast cancer and prostate cancer. JAMA 2009;302:1685-92.
8. Pedersen JH, Ashraf H, Dirksen A, et al. The Danish randomized lung cancer CT screening trial-overall design and results of the prevalence round. $J$ Thorac Oncol 2009;4:608-14.

9. Saghir Z, Ashraf H, Dirksen A, et al. Contamination during 4 years of annual CT screening in the Danish Lung Cancer Screening Trial (DLCST). Lung Cancer 2011:71:323-7.

10. Brodersen $\mathbf{J}$, Thorsen $\mathrm{H}$. Consequences of screening in breast cancer (COS-BC): development of a questionnaire. Scand J Prim Health Care 2008;26:251-6.

11. Brodersen J, Thorsen $\mathrm{H}$ Kreiner S. Consequences of screening in lung cancer: development and dimensionality of a questionnaire. Value Health 2010;13:601-12.

12. Pellegrino R, Viegi $G$, Brusasco $V$, et al. Interpretative strategies for lung function tests. Eur Respir J 2005:26:948-68.

13. van Klaveren RJ, Oudkerk M, Prokop M, et al. Management of lung nodules detected by volume CT scanning. N Engl J Med 2009;361:2221-9.

14. Ashraf $\mathbf{H}$, de HB, Shaker SB, et al. Lung nodule volumetry: segmentation algorithms within the same software package cannot be used interchangeably. Eur Radiol 2010;20:1878-85

15. Goldstraw P, Crowley J, Chansky K, et al. The IASLC Lung Cancer Staging Project: proposals for the revision of the TNM stage groupings in the forthcoming (seventh) edition of the TNM classification of malignant tumours. J Thorac Oncol 2007:2:706-14.

16. World Health Organisation-WHO. Cancer. 2011. http://www.who.int/ mediacentre/factsheets/fs297/en/ (accessed 12 Jan 2012).

17. Aberle DR, Adams AM, Berg CD, et al: National Lung Screening Trial Research Team. Reduced lung-cancer mortality with low-dose computed tomographic screening. N Engl J Med 2011;365:395-409.

18. Aberle DR, Berg CD, Black WC, et al. The national lung screening trial: overview and study design. Radiology 2011;258:243-53.

19. Lindell RM, Hartman TE, Swensen SJ, et al. 5-year lung cancer screening experience: growth curves of 18 lung cancers compared to histologic type, CT attenuation, stage, survival, and size. Chest 2009;136:1586-95.

20. Lopes PA, Picozzi G, Mascalchi M, et al. Design, recruitment and baseline results of the ITALUNG trial for lung cancer screening with low-dose CT. Lung Cancer 2009:64:34-40.

\section{Journal club}

\section{Helicobacter pylori infection in neonatal mice prevents allergic asthma}

In this preclinical study, the authors hypothesised that neonatal infection with an immonomodulatory pathogen such as Helicobacter pylori provides protection from allergic airway inflammation and hyper-responsiveness seen in allergic asthma.

C57BL/ 6 mice were orally infected with $H$ pylori at 6 days (neonatal) and 6 weeks (adults) after birth. Infected and non-infected mice underwent ovalbumin sensitisation followed by aerosolised ovalbumin challenge 4 weeks later. Infected mice as compared with non-infected mice showed significant reduction in airway hyper-responsiveness to methacholine challenge. This reduced inflammatory response was indicated by low eosinophils and interleukin 5 in bronchoalveolar lavage fluid and reduced infiltration of Th2 and Th17 cells. These changes were absent in adult infected mice indicating that only early life exposure to $H$ pylori infection confers protection against asthma in mouse models.

The authors explained the immunological process by carrying out further tests, which showed that ' $H$ Pylori-mediated asthma protection' in neonatally infected mice is due to the suppressive activity of $\mathrm{CD}^{+}{ }^{+} \mathrm{FoxP} 3^{+}$Tregs and the presence of semimature dendritic cells, both of which accumulate in the lungs during the inflammatory process. Based on the results of the mouse model, it is possible that allergic asthma is associated with the loss of indigenous microbial flora in the neonatal period; however, extrapolating this evidence to a human population will require more direct evidence from human studies.

- Arnold IC, Dehzad N, Reuter S, et al. Helicobacter pylori infection prevents allergic asthma in mouse models through the induction of regulatory T cells. J Clin Invest 2011;121:3088-93.

\section{Nawaid Ahmad}

Correspondence to Dr Nawaid Ahmad, ST5 Respiratory Medicine. University Hospitals North Staffordshire, Newcastle Road, Stoke-on-trent, ST4 60G, UK; navvydoc@gmail.com

Published Online First 22 September 2012

Thorax 2012;67:301. doi:10.1136/thoraxjnl-2011-201061 